Revista General de Información y Documentación ISSN: 1132-1873

https://dx.doi.org/10.5209/rgid.66970

\title{
El uso de fuentes documentales en internet para verificar las informaciones digitales: estudio de casos
}

\author{
Juan Carlos Marcos Recioํㅜ ${ }^{1}$ Concha Edo Bolós²; David Parra Valcarce ${ }^{3}$ \\ Recibido: 25 de junio de 2019 / Aceptado: 28 de noviembre de 2019
}

\begin{abstract}
La confianza solo se recuperará cuando la verdad vuelva al centro del escenario. Las instituciones deben responder a la demanda del público para proporcionar información objetiva precisa y oportuna y unirse al debate público. Los medios no pueden hacerlo solos debido a restricciones políticas y financieras.

Richard Edelman
\end{abstract}

Resumen. Hace más de un cuarto de siglo que los periódicos están buscando su hueco en la sociedad. Lo perdieron cuando la tecnología se hizo digital y facilitó la manera de crear, producir y enviar contenidos. Entre los medios digitales y los lectores no hay una relación de igualdad, ni de necesidad, ni siquiera de interés por la información, porque la mayoría la encuentran en otros sitios de Internet. La transformación tecnológica a la que se han visto sometidos es tan solo la punta del iceberg al que se enfrentan en la actualidad. Los cambios no son solo tecnológicos, sino económicos, financieros para el medio, sociales y de credibilidad. Este último está de moda entre la comunidad científica, por la gran cantidad de contenidos falsos que circulan en las redes y también en algunos periódicos. Hay que recuperar la confianza en los medios de comunicación. El presente artículo tiene como objetivo evaluar las fuentes documentales que se emplean en los periódicos digitales para verificar las informaciones. Para ello, se hace un estudio de los medios digitales españoles y la utilización de sus redes sociales.

Palabras clave: Fuentes documentales; Información de calidad; Lectores; Contenidos informativos; Noticias falsas; Periódicos digitales.

\section{[en] The use of documentary sources on the internet to verify digital information: case studies}

Abstract. For more than a quarter of a century, newspapers are looking for their place in society. They lost it when the technology became digital and facilitated the way to create, produce and send content. Between digital media and readers, there is no relationship of equality, of necessity, or even of interest in

$1 \quad$ Universidad Complutense de Madrid

E-mail: jmarcos@ucm.es

2 Universidad Complutense de Madrid

E-mail: conchaed@ccinf.ucm.es

3 Universidad Complutense de Madrid

E-mail: davidparra@ccinf.ucm.es 
information, because most of them find it on other Internet sites. The technological transformation to which they have been subjected is only the tip of the iceberg they face today. The changes are not only technological, but economic, financial for the environment, social and credibility. The latter is in fashion among the scientific community, for the large number of false content circulating in the networks and also in some newspapers. We must regain confidence in the media. This article aims to evaluate the documentary sources used in digital newspapers to verify information. For this, a study of the Spanish digital media and the use of their social networks is made.

Keywords: Documentary sources; Quality information; Readers; Informative content; Fake news; Digital newspapers.

Sumario. 1. Introducción. 2. Análisis de la transformación tecnológica de los periódicos digitales. 3. Principales objetivos en la elaboración de información de calidad. 4. Metodología. 5. Resultados. 6. Conclusiones y discusiones. 7. Notas. 8. Referencias bibliográficas.

Cómo citar: Marcos Recio, J.C.; Edo Bolós, C.; Parra Valcarce, D. (2019) El uso de fuentes documentales en internet para verificar las informaciones digitales: estudio de casos, en Revista General de Información y Documentación 29 (2), 309-323.

\section{Introducción}

La estrecha relación que durante al menos dos siglos experimentaron los periódicos impresos, continuada a lo largo de varias generaciones, se fue deteriorando a finales del siglo XX. La adaptación de los contenidos informativos a los nuevos lectores de periódicos digitales no se hizo con garantías de éxito; de ahí que los periódicos perdieron poder en la sociedad y dejaron de ser su referente. La aparición de las redes sociales fue el golpe definitivo para estos medios que experimentaron como una parte importante de sus lectores, sobre todo los más jóvenes, se convertían en usuarios de las redes y se alejaban definitivamente del periódico. Otra cosa bien distinta fue la consideración de los propios nativos digitales que tuvieron que construir su audiencia compitiendo con dichas redes.

A esta situación no se llegó solo por procesos técnicos. En el caso de los periódicos digitales, la pérdida o la aceptación de nuevos y jóvenes lectores se debe a varias causas. La más importante: la información de calidad, aquella que se contrasta y que busca referentes informativos, es la que marca la línea divisoria entre buen y mal periodismo.

El objetivo principal de este artículo es descubrir como la información, que sigue utilizando fuentes documentales fiables, muestra realidades informativas creíbles. En estos tiempos, los contenidos exclusivos están perdido fuerza. Hay otros elementos que indican que la información es atractiva para los lectores. La construcción de contenidos debe contar con datos precisos. Frente a la multitud de informaciones que generan ideas falsas, el periodista selecciona los que fielmente representan la información. Además, la rapidez no la marca la competencia frente a las redes sociales, sino el reto del redactor para: crear, contrastar, evaluar, publicar y actualizar. Estos cinco pasos siguen siendo claves para dotar de credibilidad a los contenidos de los periódicos digitales. Llegar antes no significa, como en el siglo pasado, ser el mejor medio. En la actualidad, el valor del tiempo informativo es muy diferente. La mayoría de los lectores se enteran antes por un amigo y por las 
redes sociales que por los periódicos digitales. Pero, luego desean ampliar la información y acuden a un medio de comunicación en Internet. Al llegar al medio es cuando el redactor tiene que sorprenderles con una buena información, con más datos, mejor contrastados, más fiable y veraces que la mayor parte de los que se publican en las redes sociales. Entonces, el lector encuentra la diferencia y se da cuenta del valor que tienen los periódicos digitales. En ese proceso, el redactor, debe dejarse acompañar por buenas fuentes de información.

\section{Análisis de la transformación tecnológica de los periódicos digitales}

Mientras que la manera en que se preparaba, redactaba y publicaba una información en los medios impresos estaba establecida desde hace décadas, el mismo proceso en los digitales no ha llegado a consolidarse definitivamente. En este camino, los periodistas vivieron turbulencias informativas que no beneficiaban su labor. Hubo varios intentos por parte de los editores para establecer los principios que deberían acometer para la producción de contenidos multimedia: a) la información impresa y digital se publica sin cambios (aparición de los primeros periódicos digitales), b) crear una información similar a la versión impresa, c) elaborar informaciones con menos textos y más recursos para atraer sobre todo a los lectores más jóvenes, d) romper definitivamente la información que publica la versión impresa y la digital, cuando las redacciones vuelven a separarse y e) la información digital que los medios nativos contemplan como válida para atraer a sus lectores.

En los procesos de transformación tecnológica de los medios digitales hay bastante literatura científica que nos sirve para sustentar el análisis que este artículo propugna. Desde comienzo de los años 90 del siglo pasado, las redacciones se han reinventado. Han tenido experiencias de: a) disgregación cuando un número pequeño de redactores deja la versión impresa y pasa a producir contenidos para el digital y b) de integración para hacer dos productos, el impreso y el digital dentro de una redacción integrada. Uno de los precursores, que avanzó con éxito lo que podría suceder fue Deuze (2004), quien se pregunta qué es el periodismo multimedia, dentro del cual se establece el periodismo digital. Por su parte DíazNoci, (2008), Salaverría, \& Negredo, (2008), Salaverría, \& García (2008), Masip (2011), Lafuente, (2012) trabajaron sobre el ciberperiodismo, sus contenidos y las nuevas redacciones que se estaban creando en los periódicos digitales, a partir de la llamada integración entre las dos redacciones de los medios, a través de una convergencia tecnológica que implicaba la adaptación de contenidos a dos versiones, la impresa y la digital. Más reciente es la aportación sobre otro tipo de narrativas que se deben incluir en los periódicos digitales, propuesta que hizo Scolari en 2013.

Dentro de este entorno tecnológico, los resultados finales a la hora de producir contenidos deben contemplar lo que el lector desea. Nunca antes el marketing fue tan necesario para los periodistas. En los medios digitales se puede escuchar al lector, se sabe cuántos están leyendo una información, cuántos días se puede dejar 
porque continúan leyendo ese contenido que se va actualizando y también se sabe qué noticia/s no se lee/n nunca, o muy pocas veces. Mientras que por siglos el lector fue el compañero ideal de los periódicos impresos, en tiempo de la transformación tecnológica, han pasado a ser la razón única que sustenta el medio. Sin lectores, sin audiencia, lo digital no tiene razón de ser. Sobre todo, porque hubo un tiempo a comienzos del siglo XXI en el que los editores esperaban que nuevas generaciones -jóvenes nativos digitales- se incorporaran como lectores. No fue así. La llegada de las redes sociales, más atractivas y más multimedia que el propio periódico digital, que empezó a elaborar vídeos informativos para competir con las redes, no fueron suficientemente interesantes como para sumar a esos lectores.

Cabe preguntarse: ¿ ¿Se lee menos desde la llegada de los periódicos digitales? ¿El aumento de seguidores en las redes sociales disminuye la presencia de los lectores en estos periódicos? Y ¿ ¿es equiparable la información que como lectores reciben de periódicos y de redes sociales?

Contrariamente a lo que la sociedad piensa en general, cada vez se lee más. Otra cuestión es separar la lectura de libros, periódicos y revistas y redes sociales. Para ello, se toma como referente el Barómetro de Hábitos de Lectura y Compra de Libros 2017, elaborado por la Federación de Gremios de Editores de España (FGEE). Así, desde 2008 hasta 2017 se ha producido un incremento de lectores (Fig. 1) pasando de un $90,1 \%$ a un $94,7 \%$. De esos totales, el $65,8 \%$ corresponde a libros, el $74,6 \%$ a Periódicos, el $41,2 \%$ a revistas y el $56,9 \%$ a redes sociales, al menos una vez al trimestre.

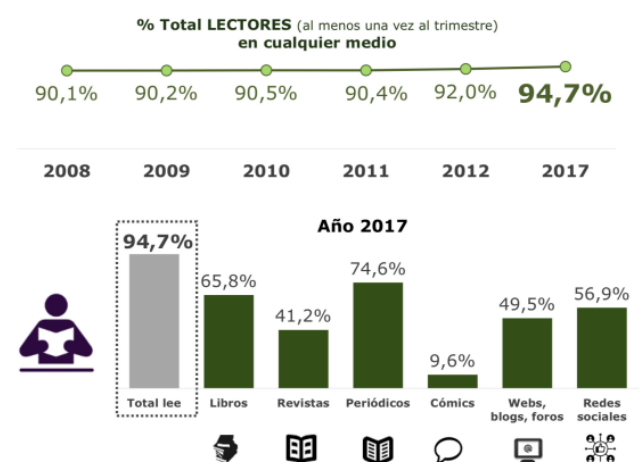

Fig. 1 Total lectores en cualquier medio

Fuente: Federación de Gremios de Editores de España (FGEE)

Con respecto a la lectura en soporte digital, el Barómetro ofrece también cifras significativas (Fig. 2): "El 76,3\% de los españoles mayores de catorce años leen algún tipo de contenido en soporte digital. Esto supone un incremento de 18 puntos con respecto a 2012. Si bien la lectura de libros en formato digital es menor que la de prensa o redes sociales, su incremento en los últimos años ha sido más significativa: ha pasado del 11,7\% de 2012 al 27,2\% en 2017. El 25\% de los libros leídos fueron en formato digital”. 


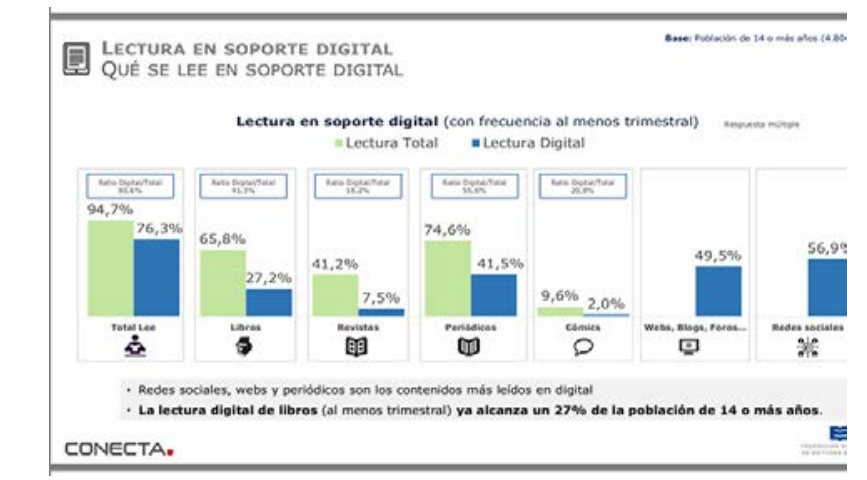

Fig. 2 Lectura en soporte digital.

Fuente: Federación de Gremios de Editores de España (FGEE)

Como se puede observar, del total de los que leen periódicos (74,6\%), el 41,5\% lo hace a través de un medio digital.

En este proceso se dividen los caminos entre los periódicos digitales que usando fuentes elaboran contenidos creíbles, sustentados en datos contrastados, con informaciones verificadas y con una visión más profesional de la información y otros contenidos que se generan en las redes sociales y que no cuentan con la garantía de una buena redacción.

Los editores no han sabido o no han podido encontrar una respuesta a esta pregunta que formuló Pfauth en 2017: “¿La vieja práctica de informar a los lectores a través de una avalancha de sucesivas noticias sigue teniendo sentido?” Sobre todo, cuando los tiempos presentes requieren de actualizar los contenidos para que los lectores acudan varias veces al medio digital. La respuesta a las preguntas anteriores se establece en esta idea: "Hoy trabajamos en línea y utilizamos más imágenes y vídeos, pero seguimos haciendo lo que siempre hemos hecho: informar al público mediante la publicación de artículos nuevos todos los días. Cuando sucede algo, escribimos una historia. Cuando sucede algo más, escribimos una nueva historia” (Pfauth 2017). Y eso es lo que diferencia el periodismo digital del que ofrecen las redes sociales, historias bien contadas que a su vez generan nuevas historias.

\section{Principales objetivos en la elaboración de información de calidad}

Son tiempos convulsos los que viven los periódicos digitales. También el resto de medios. Como lo son también los contenidos que ofrecen las redes sociales para competir con ellos. Dudas, manipulaciones, informaciones falsas, manejo de algoritmos y bots para hacer cambiar de parecer a los ciudadanos que antes sí confiaban en los medios. ¿Qué está pasando en realidad? ¿Por qué se cree más a lo que publican algunas redes sociales que a los propios medios? Una primera piedra para retomar la confianza en los medios es volver a informar con rigor. Esto 
implica mantener las redacciones y los centros de documentación y tener tiempo para elaborar las noticias. Por eso, resulta difícil de explicar que en un mismo día, las redacciones de varios medios terminen con un porcentaje muy alto de despedidos y otras estén creando nuevas vías para dotar de información dentro de la sección de tecnología.

Según informa la revista Forbes, la Corporación Meredith despidió a casi 300 empleados, en su mayoría de Time Inc.: "Los trabajadores que cubren la señalización de Time Inc. Meredith Corp., que adquirió Time Inc. en enero de 2018 por 1.850 millones de dólares, terminará despidiendo entre 200 y 300 empleados” (Fletcher, 2018). Otro tanto sucedió en la redacción de Chicago Tribune, con nuevos despidos: "Los periódicos de todo el país han sufrido despidos en los últimos días. Y ayer, fue el turno del Chicago Tribune. Aunque el número de despidos no fue enorme, las ansiedades son altas en la empresa matriz de ese medio, Tronc" (Folkenflik, 2018). La parte buena procede de The Telegraph quien acaba de crear una nueva sección de tecnología con 15 empleados: "The Telegraph ha lanzado una nueva tecnología vertical: Tecnología de Inteligencia. Cubrirá revisiones de dispositivos y noticias, compañías de tecnología, desarrollos tecnológicos en áreas como criptomonedas y realidad aumentada, los efectos políticos y sociales de la tecnología, además de la escena de inicio en el Reino Unido" (Southern, 2018).

No puede haber información de calidad cuando la mayoría de las redacciones están recortando sus redacciones, porque con menos periodistas y con más temas para tratar, no se puede mejorar los contenidos. Lo mismo se puede decir de los apoyos documentales que reciben los periodistas a la hora de preparar sus contenidos digitales. Menos documentalistas también equivale a informaciones más lentas pero con menos garantías. La pregunta clave sería: ¿Es mejor llegar antes sin garantías de que la información esté contrastada? $\mathrm{O}$ ¿Es preferible no crear informaciones sesgadas y ofrecer los contenidos una vez están contrastados? Caben respuestas intermedias porque ninguna de las dos opciones es buena.

Uno de los grandes logros que fue resolviendo la tecnología incipiente del siglo $\mathrm{XX}$ era llegar a los lectores cuanto antes mejor. Esta máxima periodística sigue siendo válida, pero ya casi es imposible ofrecer exclusivas y lo que buscan los lectores digitales son contenidos fiables y creíbles frente a lo que leen en redes sociales y de las que desconfían. De ahí, la importancia que tiene visualizar las fuentes de información que emplean los periodistas para su creación.

Resulta difícil saber cuál es el punto de partida cuando el periodista acuciado por el tiempo y por los diversos temas que cubre a la vez no puede detenerse ni profundizar en los contenidos. Es hora de buscar apoyos en el centro de documentación y tomar datos de otras fuentes. Cuando a los periodistas se les pregunta sobre los apoyos que necesitan para su trabajo responden: "La mayoría afirman que los temas surgen de sus contactos con fuentes personales, seguidas de la información que procede de fuentes públicas como oficina de prensa, observatorios o instituciones" (Informe APM, 2017).

Cuando se trata de confianza en los medios, ésta desciende de manera significativa. Así lo atestigua el Edelman Trust Barometer cuando indica que más 
del 60\% de los ciudadanos encuentran dificultades para dirimir si una noticia ha sido generada por un medio de comunicación respetado. Y lo que es peor, un 59\% se muestra incapaz de identificar la verdad. Estos datos han llevado al director del Barometer a presentar una realidad preocupante: "La gente ha perdido la confianza en los canales de comunicación y en las fuentes de noticias. Ya no estamos dispuestos a creer la información que se nos presenta, incluso cuando proviene de los más cercanos a nosotros. Estamos enfrentando una crisis de desinformación sin precedentes” (Edelman 2018).

Para avanzar en el camino hacia la verdad ya hay organismos que tratan de poner freno a las informaciones falsas mediante la verificación, como es el caso de la Red Internacional de Verificación de Datos, del Instituto Poynter y la que han generado algunos medios de comunicación para uso interno. En todo caso, se preguntaba Otero: ¿Qué hace que una fuente de noticias sea buena? Porque en la respuesta está mejorar la calidad de la información: "En el pasado, los programas nacionales de noticias vespertinos, los programas locales de noticias nocturnas y las portadas de los periódicos impresos estaban dominados por reportajes de hechos. Ahora, sin embargo, muchas fuentes que la gente considera como "fuentes de noticias” en realidad están dominadas por análisis y artículos de opinión” (Otero 2017). Quizás, la solución sea un regreso al pasado para volver a retomar la manera en que se redacta la información.

\subsection{Principales objetivos}

El objetivo principal de esta investigación es descubrir como la información que sigue usando fuentes documentales fiables muestra realidades informativas más creíbles. El manejo de dichas fuentes, su frecuencia y el apoyo en la información que se genera a través de las redes sociales, marcan el proceso de creación de contenidos de los periodistas. El texto muestra una realidad en la que viven cada día los redactores, a la que se enfrentan con gran cantidad de bulos que no siempre contribuyen a clarificar la situación informativa. Otros objetivos importantes que contempla esta investigación son: a) la importancia que tiene el centro de documentación para los redactores, b) los fondos que tienen, su actualización y c) las bases de datos que con mayor frecuencia emplean. Además, otros medios extranjeros que toman como referente y el valor informativo que dan a las redes sociales y a los blogs.

\subsection{Formulación de los datos}

Un acercamiento a los redactores de medios digitales es la mejor manera para conocer cómo están elaborando sus contenidos. Se utiliza, por tanto, una metodología consistente en analizar las principales fuentes documentales que se vienen empleando en algunos periódicos digitales. A través de una encuesta cualitativa se pretende obtener el número y tipo de fuentes que los principales periódicos digitales emplean para la elaboración de su información. 
Para ello, se envió una encuesta a 50 periodistas del ámbito digital, de los 10 medios digitales que según Comscore MMX tienen más usuarios únicos mensuales: El País, El Mundo, La Vanguardia, Abc; El Confidencial; 20 minutos, OK Diario, El Periódico, El Diario y El Español. Se obtuvieron 41 respuestas.

\section{Metodología}

Las fuentes de información son el referente obligado para mejorar las informaciones digitales. Para demostrarlo en este artículo, se empleó una metodología cuantitativa que iba a permitir conocer dos aspectos importantes a través de una encuesta: el funcionamiento de los centros de documentación y el uso que hacían de dichas fuentes, y el valor real de uso de las redes sociales como apoyo documental a los contenidos de los periodistas. Para ello, se seleccionaron los medios digitales que tienen más usuarios únicos y tras analizar sus informaciones se procedió a preparar una encuesta que separa las preguntas relacionadas con el centro de documentación, de las propias de la redes sociales, con estos resultados.

\section{Resultados}

\subsection{Generales, relativos a los Centros de Documentación}

El primer propósito para entender el valor de las fuentes de información es valorar el origen de las informaciones, especialmente las que provienen del centro de documentación y o empresas externas. En el primer caso, el 80,5\% de los encuestados (34 respuestas) dijeron sí y el 19,5\% (7 respuestas) fueron no. En cuanto a las empresas externas las respuestas fueron más diversas: Sin centro respondieron 13 encuestados y 2 emplean empresas externas. El resto, usan consultorías y/o el centro interno. Con respecto al volumen de fuentes, la mejor cifra es precisamente la más alta (tabla 1), con un 73,5\% que tienen más de 5.000 documentos, fuentes $\mathrm{y} / \mathrm{o}$ libros.

Tabla 1. Volumen de fuentes/libros/documentos del Centro de Documentación

\begin{tabular}{|l|c|c|}
\hline Menos de mil & $8,8 \%$ & 3 \\
\hline Entre 1.000 y $3.000 \mathrm{mil}$ & $5,9 \%$ & 2 \\
\hline Entre 3.000 y $5.000 \mathrm{mil}$ & $11,8 \%$ & 4 \\
\hline Más de 5.000 & $73,5 \%$ & 25 \\
\hline \multicolumn{2}{|c|}{ Total: 34 respuestas. Fuente: Elaboración propia ${ }^{4}$. }
\end{tabular}

En este artículo se pedía a los periodistas que valoraran entre el centro de documentación y los diferentes repertorios, con resultados favorables al primero

4 Todas las tablas y gráficos son de elaboración propia. 
con un 78,9\% y un 21,1\% para los repositorios. Además, el grado de satisfacción de dichos centros está muy bien valorado, con los siguientes resultados: Un 52,9\% (13 respuestas) respondió que óptimo, un 29,4\% (10 respuestas) aceptable, un $17,6 \%$ (6 respuestas) mejorable y 7 periodistas no contestaron. También a los que trabajan en la versión digital se les cuestiona sobre la importancia que tiene en tiempos de las redes sociales este tipo de centros, sobre todo porque la información se actualiza constantemente. Respondieron un $65,9 \%$ que sí, en determinados casos un $17,1 \%$, en Internet se encuentra todo un $9,8 \%$ y no un $7,2 \%$. La última pregunta más abierta, con respecto a otro tipo de almacenamientos empleados, una parte importante de los periodistas señaló que trabajan con una intranet corporativa que da acceso a todo el diario digitalizado y documentos primarios de alto valor documental como elecciones, cronologías, efemérides, etc., o la base de datos Quay, o una intranet corporativa donde se puede consultar El Periódico de Catalunya desde su inicio en 1978 hasta la actualidad. Entre las respuestas se producen algunas limitaciones a ciertas secciones y/o a algunos redactores. Y no falta entre las respuestas la nube como almacenamiento conectado.

\subsection{Generales, relativos a los Redes sociales}

Si los medios digitales sienten la presión cada vez mayor de las redes sociales, este trabajo quería valorar la parte de información que los periodistas toman de las propias redes, especialmente Twitter (Tabla 2), Facebook (Tabla 3) e Instagram (Tabla 4), dejando la última pregunta abierta para conocer qué otras redes emplean como fuente de información. En Twitter, un 50\% de los periodistas encuestados emplea como fuente de información más de un $10 \%$, seguido de un $27,5 \%$ que lo hacen entre un $2 \%$ y un $5 \%$ y un $12,5 \%$ entre un $8 \%$ y un $10 \%$, dejando solo el $10 \%$ entre un $5 \%$ y un $8 \%$. Por el contrario, en Facebook, los porcentajes cambian significativamente, siendo la cifra más alta, un $60 \%$ los que emplean esta red social entre un $2 \%$ y un $5 \%$, seguida del $20 \%$ entre un $8 \%$ y un $10 \%$, un $15 \%$ más de un $10 \%$ y tan solo un $5 \%$ entre un $5 \%$ y un $8 \%$.

Tabla 2. \% diario de Twitter

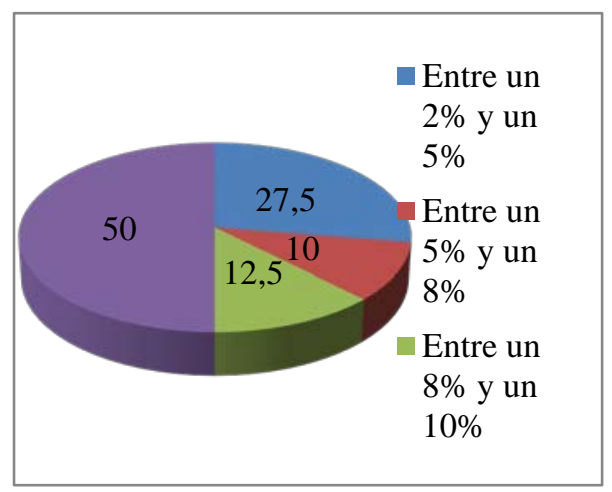

Tabla 3. \% diario de Facebook

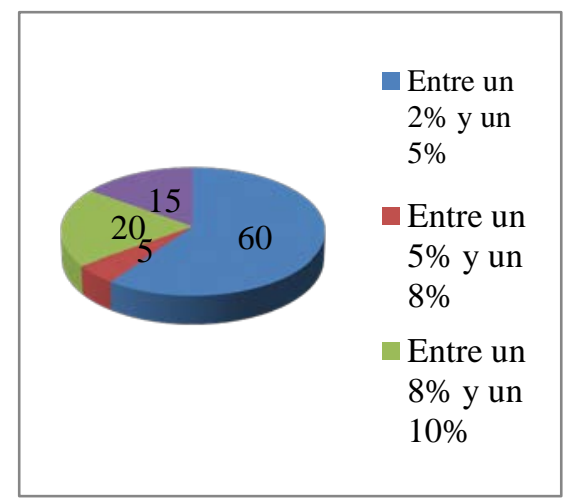


Tabla 4. \% diario de Facebook Instagram

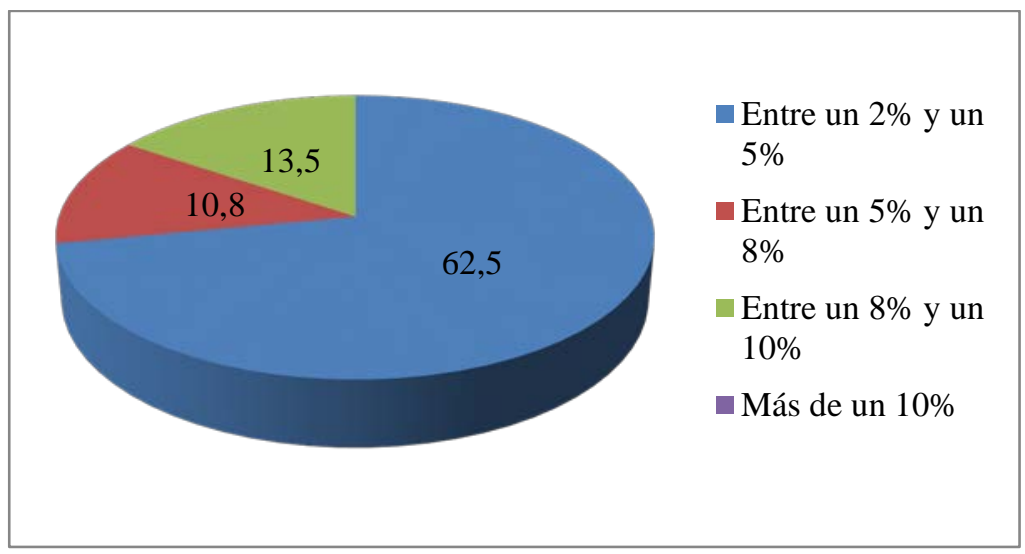

Por su parte, en Instagram asciende aún más el porcentaje más pequeño de uso, ya que el $62,5 \%$ lo emplea como fuente de información entre un $2 \%$ y un $5 \%$, seguido del $13,5 \%$ que lo hace entre un $8 \%$ y un $10 \%$ y un $10,8 \%$ entre un $5 \%$ un $8 \%$. En definitiva, Twitter supera a las dos redes analizadas, quedando en segundo lugar Facebook y luego Instagram.

La última pregunta, abierta, incluía la opción de contestar sobre otras redes sociales que los periodistas emplearan como fuente de información. Las respuestas variadas se puede resumir en las siguiente: LinkedIn, Pinterest, Youtube, Snapchat, Tumblr, Reddit, Meneame, Canales de Telegram, Vipter, etc. De las 27 respuestas obtenidas, 20 dijeron que no utilizaban otras redes y algún periodista señaló como aspecto destacable: "Linkedin. Sólo utilizo las redes sociales como fuente de información si no existe otra fuente, o para reconfirmar, por repetición, una información”. Por tanto, las redes sociales como fuente de información figuran en un lugar poco destacable para los periodistas.

\subsection{Fuentes específicas}

En este apartado se pretende descubrir qué tipo de fuentes, bases de datos españolas e internacionales, así como agencias y medios de comunicación emplean los redactores a la hora de crear su información. En primer lugar se ofrece en respuesta múltiple la posibilidad de responder a las fuentes que emplean con más frecuencia (Tabla 5). El apartado otras, con un $72,5 \%$ es el más empleado, seguido de diccionarios y directorios. 
Tabla 5. Fuentes que usan con más frecuencia

\begin{tabular}{|c|c|c|}
\hline & respuesta & $\%$ \\
\hline Anuarios & 9 & 22,5 \\
\hline Bibliografía y libros & 18 & 45 \\
\hline Diccionarios & 21 & 52.5 \\
\hline Directorios & 17 & 42,5 \\
\hline Guías & 16 & 40 \\
\hline Otros & 29 & 72,5 \\
\hline
\end{tabular}

En el apartado de base de datos, tanto españolas como específicas (tablas 6 y 7), las bases propias del medio, entre ellas las del propio periódico, suponen el 67,6\% con una amplia mayoría de uso, solo superado por el BOE con un $73 \%$. El resto con una media del 20 por ciento apenas son significativas. Lo mismo sucede en las bases de datos específicas, donde Google News marca la gran diferencia con un $64,9 \%$, seguida del $59,5 \%$ de otras.

Tabla 6. Bases de datos españolas

\begin{tabular}{|l|c|c|}
\hline & respuesta & $\%$ \\
\hline Aranzadi & 9 & 24,3 \\
\hline BOE & 27 & 73 \\
\hline ISBN & 8 & 21,6 \\
\hline CSIC & 9 & 24,3 \\
\hline Baratz & 3 & 8,1 \\
\hline Base propias del medio & 25 & 67,6 \\
\hline
\end{tabular}

Tabla 7. Bases de datos específicas

\begin{tabular}{|l|c|l|}
\hline & respuesta & $\%$ \\
\hline Google News & 24 & 64,9 \\
\hline Lexis-Nesis & 4 & 10,8 \\
\hline Broadcast News & 6 & 16,2 \\
\hline ProQuest & 6 & 16,2 \\
\hline Current content & 5 & 13,5 \\
\hline Econtit & 7 & 18,9 \\
\hline Factiva & 8 & 21,6 \\
\hline Dare database & 2 & 5,4 \\
\hline Otras & 22 & 59,5 \\
\hline
\end{tabular}

Con respecto a la información que obtienen de las agencias, las españolas son las que figuran con más demanda y de ellas, EFE con un 95,1\%, seguida de Europa Press, un 92,7\%, Reuters en tercer lugar con un 73,2\%. Luego France Press un 
56,1\% y Associated Press y Otras con un 46,3\% cada una. El resto, incluida la española Servimedia, supone algo más de un $20 \%$.

Los propios medios de comunicación tanto españoles como de otros países sirven como referente informativo para algunos temas internacionales, pero también con incidencia en España. En el caso de los medios españoles (Tabla 8) y en los extranjeros (tabla 9), El País es el que mejor valoración obtiene con un 70,3\%, seguido de El Mundo, con un 64,9\%. En los medios de fuera, el apartado Otros suma la cifra más importante con un 67, 6\%, siendo The New York Times con el 59,5\% el más utilizado como fuente, seguido del The Washington Post con un 54,1\%.

Tabla 8. Medios españoles como fuente.

\begin{tabular}{|l|c|c|}
\hline & Respuesta & $\%$ \\
\hline El País & 26 & 70,3 \\
\hline El Mundo & 24 & 64,9 \\
\hline Abc & 20 & 54,1 \\
\hline La Razón & 10 & 27 \\
\hline La vanguardia & 21 & 56,8 \\
\hline La voz de Galicia & 12 & 32,4 \\
\hline $\begin{array}{l}\text { El periódico de } \\
\text { Cataluña }\end{array}$ & 16 & 43,2 \\
\hline El Correo & 14 & 37,8 \\
\hline Otros & 21 & 56,8 \\
\hline
\end{tabular}

Tabla 9. Medios extranjeros como fuente

\begin{tabular}{|l|c|c|}
\hline & respuesta & $\%$ \\
\hline New York Times & 22 & 59,5 \\
\hline The Washington Post & 20 & 54,1 \\
\hline The Wall Street Journal & 9 & 24,3 \\
\hline The Economist & 13 & 32,4 \\
\hline The Financial Times & 15 & 40,5 \\
\hline The Telegraph & 8 & 21,6 \\
\hline Frantfuter Allgemeine & 6 & 16,2 \\
\hline Die Welt & 3 & 8,1 \\
\hline Le Monde & 20 & 54,1 \\
\hline Otros & 25 & 67,6 \\
\hline
\end{tabular}

El valor de algunas informaciones que vienen apareciendo en determinados blogs, fue la excusa para preguntar a los periodistas sobre el porcentaje diario que empleaban para hacer información tomándolos como referente. Así, 23 periodistas respondieron que entre un $2 \%$ y un $5 \%$; 10 , entre un $5 \%$ y un $8 \%$, 2 entre un $8 \%$ y un $10 \%$ y 3 lo hacían con más de un 10\%. Lo que se traduce en que los blogs, apenas forman parte de las fuentes que los redactores usan para crear sus contenidos diarios. 


\section{Conclusiones y discusiones}

La mayor parte de la sociedad considera que está lo suficientemente bien informada. La realidad indica que no es cierto. Al menos, a tenor de los resultados de este estudio. La introducción de la tecnología en los medios, las redes sociales y las más variadas maneras de presentar la información está llevando al ciudadano a contenidos que no se ajustan a la verdad. En tiempos de la postverdad, el compromiso de los periodistas es ajustarse a la información veraz, objetiva y pertinente. Por eso, este artículo ofrece herramientas, fuentes de información y contenidos a través de los cuales los periodistas pueden crear contenidos más fiables. Los resultados del mismo justifican esa relación que existe entre el uso de fuentes ya establecidas, clásicas en la documentación, y las nuevas maneras de conseguir información rápida, pero en ocasiones sin contrastar. Sin embargo, se percibe una mejora en los contenidos cuando se accede de manera ágil a algunas propuestas que están en las redes sociales y que permiten al periodista avanzar hacia una propuesta informativa que no había contemplado. En definitiva, ser y estar en la información para abrir caminos a través de las redes sociales como Twitter, Facebook o Instagram.

La necesidad cada vez mayor por obtener informaciones verídicas, tras el ciclón de noticias falsas, los movimientos para influir en las decisiones políticas y la utilización de bots y máquinas que repiten mensajes sin cesar, requiere que los periodistas estén cada día más atentos a los mensajes que circulan por las redes sociales. Para ello, disponer de un centro de documentación propio, como se ha visto en los resultados, donde corroborar la información, es el reto y la necesidad que deben asumir cada día. El problema que se plantea: las redacciones y los centros de documentación están siendo recortadas cada día. También influye la manera en que el redactor sigue varias noticias en un mismo momento. Además, el nivel de lectura está mejorando, pero aún hay margen.

Los resultados de este estudio nos permiten precisar si la información que emplea fuentes documentales es más o menos fiable y atractiva para los lectores. En este sentido, la mayoría de los periodistas sigue apostando por trabajar con las fuentes que tiene en su propio medio. Un 73,5\% respondió que su centro dispone de un volumen superior a 5.000 documentos. Dentro de las fuentes que emplean fuera del ámbito Otros, tanto directorios, como diccionarios son los más recurrentes. De las bases de datos el BOE sigue siendo una fuente imprescindible y de las de fuera Google News. Los propios periódicos digitales suponen un referente para los periodistas. En este sentido, El País y el Mundo son los mejor valorados en España y fuera son The New York Times, The Washington Post y Le Monde.

Con respecto a las redes sociales y a su uso como fuente de información, solo Twitter sabe bien referenciada, ya que los periodistas acuden a ella en más de un $10 \%$ a buscar la información, mientras que Facebook e Instagram presentan cifras más bajas. Hay otras redes que también se contemplan como LinkedIn, Pinterest Youtube, Snapchat, etc. Por tanto, las fuentes de información dentro del propio medio siguen siendo la garantía para verificar las informaciones que cada día se publican en los medios digitales. 


\section{Notas}

Este artículo forma parte de los trabajos llevados a cabo en el marco del proyecto de investigación "Claves para la redefinición y supervivencia del periodismo y retos en la era post-PC. Medios emergentes, nuevas narrativas, agregadores, robots, multiscreen, first mobile, apps” (Ref. CSO2016-79782-R).

\section{Referencias bibliográficas}

APM (2017). Informe Anual de la Profesión Periodística. 16/03/2018. $<$ www.apmadrid.es/publicaciones/informe-anual-de-la-profesion/> [Consulta: 01/05/2018]

Deuze, M. (2004). What is multimedia journalism? Journalism Studies, 5 (2), 139-152.

Díaz-Noci, J. (2008.). Definición teórica de las características del ciberperiodismo: elementos de comunicación digital. Doxa Comunicación: revista interdisciplinar de estudios de comunicación y ciencias sociales, 6, 53-91.

Edelman, R. (2018). Edelman Trust Barometer. 21/03/2018, de Edelman Trust. < www.edelman.com/trust-barometer> [Consulta: 15/05/2018]

Federación de Gremio de Editores de España (2018). Hábitos de Lectura y Compra de Libros en España 2017. 14/03/2018 <http://federacioneditores.org/img/documentos/ HabitosLecturaCompraLibros2017.pdf> [Consulta: 01/06/2018]

Folkenflik, D. (2018). Layoffs Hit 'Chicago Tribune' Newsroom. 16/03/2018. < www.npr. org/2018/03/16/594199585/layoffs-hit-chicago-tribune-newsroom?t=1561395567531> [Consulta: 20/05/2018]

Fletcher, P. (2018). Meredith Corp. To Lay Off 200 To 300 Employees, Mostly From Former Time Inc. 21/03/2018 <www.forbes.com/sites/paulfletcher /2018/03/18/meredith-inc-to-lay-off-200-to-300-employees-mostly-from-former-timeinc/\#4afa97a626c2> [Consulta: 24/05/2018]

Lafuente, G. (2012). Las nuevas redacciones y el periodismo con futuro.11/02/2018. <www.apmadrid.es/wp-content/uploads/2012/06/60-64\%20REDACCIONES\%20DEL \%20FUTURO(2).pdf $>$ [Consulta: 24/05/2018]

Masip, P. (2011). Integración de redacciones en los medios locales y comarcales catalanes. Estudios Sobre el Mensaje Periodistico, 17.(1), 167-184.

Ministerio de Educación, Cultura y Deporte (2017). Barómetro de Hábitos de Lectura y Compra de Libros 2017. 24/03/2018. Plan de Fomento de la lectura 2017-2020 < http://fomentodelalectura.mecd.gob.es/actualidad/noticias/Bar-metro-2017.html> [Consulta: 22/06/2018]

Otero, V. (2017). What Makes A News Source Good?. 11/02/2018. <www.allgenera lizationsarefalse.com/the-chart-version-3-0-what-exactly-are-we-reading/> [Consulta: 22/06/2018]

Pfauth, E. J. (2017). Publishing less to give readers more. 19/03/2018. < www.niemanlab. org/2017/12/publishing-less-to-give-readers-more/> [Consulta: 04/04/2018]

Salaverría, R.; Negredo, S. (2008). Periodismo integrado: convergencia de medios y reorganización de redacciones. Barcelona. Editorial Sol 90.

Salaverría, R.; Negredo, S. (2008). La convergencia tecnológica en los medios de comunicación: retos para el periodismo. 16/03/2018. Trípodos.< www.raco.cat/ index.php/tripodos/article/viewFile/118910/154114> [Consulta: 12/05/2018]

Scolari, C. A. (2013). Narrativas transmedia. Barcelona: Deusto. 
Southern, L. (2018). The Telegraph launches new tech section with 15 staffers. 19/03/2018. https://digiday.com/media/telegraph-launches-new-tech-section-15-staffers/?utm_ source=Pew+Research+Center\&utm_campaign $=001$ b16586d-EMAIL_CAMPAIGN_ 2018_03_20\&utm_medium $=$ email\&utm_term $=0$ _3e953b9b70-001b16586d-399360629 [Consulta: 22/06/2018] 